\title{
On indicated coloring of some classes of graphs
}

\author{
P. Francis ${ }^{1}$, S. Francis Raj ${ }^{2}$ and M. Gokulnath ${ }^{3}$ \\ Department of Mathematics, Pondicherry University, Puducherry-605014, India. \\ 1: selvafrancis@gmail.com ${ }^{2}$ : francisraj_s@yahoo.com ${ }^{3}$ : gokulnath.math@gmail.com
}

\begin{abstract}
Indicated coloring is a type of game coloring in which two players collectively color the vertices of a graph in the following way. In each round the first player (Ann) selects a vertex, and then the second player (Ben) colors it properly, using a fixed set of colors. The goal of Ann is to achieve a proper coloring of the whole graph, while Ben is trying to prevent the realization of this project. The smallest number of colors necessary for Ann to win the game on a graph $G$ (regardless of Ben's strategy) is called the indicated chromatic number of $G$, denoted by $\chi_{i}(G)$. In this paper, we obtain structural characterization of connected $\left\{P_{5}, K_{4}\right.$, Kite, Bull $\}$-free graphs which contains an induced $C_{5}$ and connected $\left\{P_{6}, C_{5}, K_{1,3}\right\}$-free graphs that contains an induced $C_{6}$. Also, we prove that $\left\{P_{5}, K_{4}\right.$, Kite, Bull $\}$-free graphs that contains an induced $C_{5}$ and $\left\{P_{6}, C_{5}, \overline{P_{5}}, K_{1,3}\right\}$ free graphs which contains an induced $C_{6}$ are $k$-indicated colorable for all $k \geq \chi(G)$. In addition, we show that $\mathbb{K}\left[C_{5}\right]$ is $k$-indicated colorable for all $k \geq \chi(G)$ and as a consequence, we exhibit that $\left\{P_{2} \cup P_{3}, C_{4}\right\}$-free graphs, $\left\{P_{5}, C_{4}\right\}$-free graphs are $k$-indicated colorable for all $k \geq \chi(G)$. This partially answers one of the questions which was raised by A. Grzesik in [8].
\end{abstract}

Key Words: Game chromatic number, Indicated chromatic number, $P_{5}$-free graphs. 2000 AMS Subject Classification: 05C75

\section{Introduction}

All graphs considered in this paper are simple, finite and undirected. For any positive integer $k$, a proper $k$-coloring of a graph $G$ is a mapping $c: V(G) \rightarrow\{1,2, \ldots, k\}$ such that for any two adjacent vertices $u, v \in V(G), c(u) \neq c(v)$. A graph is said to be $k$-colorable if it admits a proper $k$-coloring. The chromatic number $\chi(G)$ of a graph $G$ is the smallest $k$ such that $G$ 
is $k$-colorable. In this paper, $P_{n}, C_{n}$ and $K_{n}$ respectively denotes the path, the cycle and the complete graph on $n$ vertices. For $S, T \subseteq V(G)$, let $\langle S\rangle$ denote the subgraph induced by $S$ in $G$ and let $[S, T]$ denote the set of all edges with one end in $S$ and the other end in $T$. $[S, T]$ is said to be complete if every vertex in $S$ is adjacent with every vertex in $T$. For any graph $G$, let $\bar{G}$ denote the complement of $G$.

Let us recall some of the definitions which are required for this paper. Let $\mathcal{F}$ be a family of graphs. We say that $G$ is $\mathcal{F}$-free if it contains no induced subgraph which is isomorphic to a graph in $\mathcal{F}$. For two vertex-disjoint graphs $G_{1}$ and $G_{2}$, the join of $G_{1}$ and $G_{2}$, denoted by $G_{1}+G_{2}$, is the graph whose vertex set $V\left(G_{1}+G_{2}\right)=V\left(G_{1}\right) \cup V\left(G_{2}\right)$ and the edge set $E\left(G_{1}+G_{2}\right)=E\left(G_{1}\right) \cup E\left(G_{2}\right) \cup\left\{x y: x \in V\left(G_{1}\right), y \in V\left(G_{2}\right)\right\}$. In this paper, we write $H \sqsubseteq G$ if $H$ is an induced subgraph of $G$. Next, the coloring number of a graph $G$, denoted by $\operatorname{col}(G)$, is defined by $\operatorname{col}(G)=1+\max _{H \subseteq G} \delta(H)$. By Szekeres-Wilf's inequality, $\chi(G) \leq \operatorname{col}(G)$.

A game coloring of a graph is a coloring of the vertices in which two players Ann and Ben are alternatively coloring the vertices of the graph $G$ properly by using a fixed set of colors $C$. The first player Ann is aiming to get a proper coloring of the whole graph, where as the second player Ben is trying to prevent the realization of this project. If all the vertices are colored then Ann wins the game, otherwise Ben wins (that is, at that stage of the game there appears a block vertex. A block vertex means an uncolored vertex which has all colors from $C$ on its neighbors). The minimum number of colors required for Ann to win the game on a graph $G$ irrespective of Ben's strategy is called the game chromatic number of the graph $G$ and it is denoted by $\chi_{g}(G)$. There has been a lot of papers on game coloring. See for instance, [9, 15, 18, 19]. The idea of indicated coloring was introduced by A. Grzesik in [8] as a slight variant of the game coloring in the following way: in each round the first player Ann selects a vertex and then the second player Ben colors it properly, using a fixed set of colors. The aim of Ann as in game coloring is to achieve a proper coloring of the whole graph $G$, while Ben tries to "block" some vertex. The smallest number of colors required for Ann to win the game on a graph $G$ is known as the indicated chromatic number of $G$ and is denoted by $\chi_{i}(G)$. Clearly from the definition we see that $\omega(G) \leq \chi(G) \leq \chi_{i}(G) \leq \Delta(G)+1$. For a graph $G$, if Ann has a winning strategy while using $k$ colors, then we say that $G$ is $k$-indicated colorable.

In [19], X. Zhu has asked the following question for game coloring. Whether increasing the number of colors will favor Ann? That is, if Ann has a winning strategy using $k$ colors, will Ann have a winning strategy using $k+1$ colors? The same question was asked by A. Grzesik for indicated coloring. The question can be equivalently stated as "Whether $G$ is $k$-indicated 
colorable for every $k \geq \chi_{i}(G)$ ". He also showed by an example that the increase in number of colors does not make life simple for Ann rather it makes it much harder. There has been already some partial answers to this question. For instance in [7, 13], R. Pandiya Raj et.al. have shown that chordal graphs, cographs, complement of bipartite graphs, $\left\{P_{5}, K_{3}\right\}$-free graphs, $\left\{P_{5}\right.$,paw $\}$-free graphs, and $\left\{P_{5}, K_{4}-e\right\}$-free graphs are $k$-indicated colorable for all $k \geq \chi(G)$. In addition, M. Lasoń in [11] has obtained the indicated chromatic number of matroids. In this paper, we obtain structural characterization of connected $\left\{P_{5}, K_{4}\right.$, Kite, Bull $\}$ free graphs which contains an induced $C_{5}$ and connected $\left\{P_{6}, C_{5}, K_{1,3}\right\}$-free graphs which contains an induced $C_{6}$. Also, we prove that $\left\{P_{5}, K_{4}, K i t e, B u l l\right\}$-free graphs that contains an induced $C_{5}$ and $\left\{P_{6}, C_{5}, \overline{P_{5}}, K_{1,3}\right\}$-free graphs which contains an induced $C_{6}$ are $k$-indicated colorable for all $k \geq \chi(G)$. In addition, we show that $\mathbb{K}\left[C_{5}\right]$, the complete expansion of $C_{5}$, is $k$-indicated colorable for all $k \geq \chi(G)$ and as a consequence, we exhibit that $\left\{P_{2} \cup P_{3}, C_{4}\right\}$ free graphs, $\left\{P_{5}, C_{4}\right\}$-free graphs are $k$-indicated colorable for all $k \geq \chi(G)$.

Notations and terminologies not mentioned here are as in [17].

\section{Structural characterization of some free graphs and their indicated coloring}

In [3], it has been shown that the game chromatic number of a bipartite graph can be arbitrarily large when compared to the chromatic number which is equal to 2. But while considering the indicated chromatic number of a bipartite graph $G$, A. Grzesik in [8] has shown that $\chi_{i}(G)=2$.

Theorem $2.1([8])$ Every bipartite graph is $k$-indicated colorable for every $k \geq 2$.

Next, let us recall the definition of complete expansion and independent expansion of a graph $G$. Let $G$ be a graph on $n$ vertices $v_{1}, v_{2}, \ldots, v_{n}$, and let $H_{1}, H_{2}, \ldots, H_{n}$ be $n$ vertexdisjoint graphs. An expansion $G\left(H_{1}, H_{2}, \ldots, H_{n}\right)$ of $G$ is the graph obtained from $G$ by (i) replacing each $v_{i}$ of $G$ by $H_{i}, i=1,2, \ldots, n$, and

(ii) by joining every vertex in $H_{i}$ with every vertex in $H_{j}$ whenever $v_{i}$ and $v_{j}$ are adjacent in $G$.

For $i \in\{1,2, \ldots, n\}$, if $H_{i}=K_{m_{i}}$, then $G\left(H_{1}, H_{2}, \ldots, H_{n}\right)$ is said to be a complete expansion of $G$ and is denoted by $\mathbb{K}[G]\left(m_{1}, m_{2}, \ldots, m_{n}\right)$ or $\mathbb{K}[G]$. For $i \in\{1,2, \ldots, n\}$, if $H_{i}=\overline{K_{m_{i}}}$, then $G\left(H_{1}, H_{2}, \ldots, H_{n}\right)$ is said to be an independent expansion of $G$ and is 


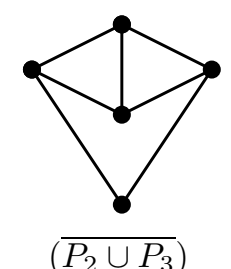

$\left(\overline{P_{2} \cup P_{3}}\right)$

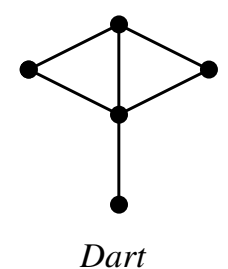

Dart

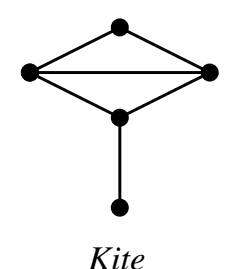

Kite

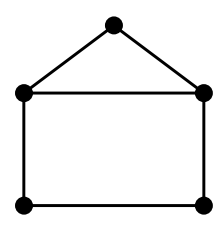

$\overline{P_{5}}$

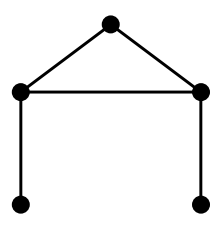

Bull

Figure 1: Some special graphs

denoted by $\mathbb{I}[G]\left(m_{1}, m_{2}, \ldots, m_{n}\right)$ or $\mathbb{I}[G]$.

In [16], D. P. Sumner studied the structural property of $\left\{P_{5}, K_{3}\right\}$-free graphs.

Theorem 2.2 (16]) Let $G$ be a $\left\{P_{5}, K_{3}\right\}$-free graph. Then each component of $G$ is either bipartite or $\mathbb{I}\left[C_{5}\right]\left(m_{1}, m_{2}, \ldots, m_{5}\right)$, where $m_{i} \geq 1$ for $i=1,2,3,4,5$.

Let us start this section with a structural characterization of a family of $P_{5}$-free graphs. The study of $P_{5}$-free graphs has been of interest for a lot of coloring parameters. For instance see, [2, 4, 6]. In this direction, we would like to consider connected $\left\{P_{5}, K_{4}, K i t e, B u l l\right\}$-free graphs that contains an induced $C_{5}$. Here, the graphs Kite and Bull are shown in Figure 1 .

Theorem 2.3 If $G$ is a connected $\left\{P_{5}, K_{4}\right.$, Kite, Bull $\}$-free graph that contains an induced $C_{5}$, then $V(G)=V_{1} \cup V_{2} \cup V_{3}$ such that (1) $\left\langle V_{2}\right\rangle$ is a complete bipartite graph with bipartitions $B$ and $S$, (2) $\left\langle V_{1} \cup V_{3}\right\rangle$ is disjoint union of $\mathbb{I}\left[C_{5}\right]^{\prime} s$ and bipartite graphs, (3) $\left[V_{1}, B\right]$ is complete, $\left[V_{1}, S\right]=\left[V_{1}, V_{3}\right]=\left[V_{3}, B\right]=\emptyset$ and (4) there exists $x^{*} \in S$ such that $\left[x^{*}, V_{3}\right]$ is complete.

Proof. Let $G$ be a connected $\left\{P_{5}, K_{4}\right.$, Kite, Bull $\}$-free graph that contains an induced $C_{5} \cong$ $\left\langle\left\{v_{0}, v_{1}, v_{2}, v_{3}, v_{4}\right\}\right\rangle=\left\langle N_{0}\right\rangle$, and let $N_{i}=\left\{x \in V(G): \operatorname{dist}\left(x, N_{0}\right)=i\right\}, i \geq 1$.

Claim 1: If $x \in N_{1}$, then $\left\langle N(x) \cap N_{0}\right\rangle \cong 2 K_{1}$ or $C_{5}$.

For $x \in N_{1}$, the possibilities for $\left\langle N(x) \cap N_{0}\right\rangle$ are $K_{1}, K_{2}, P_{3}, P_{4}, 2 K_{1}, K_{1} \cup K_{2}$ and $C_{5}$. Here (a) if $\left\langle N(x) \cap N_{0}\right\rangle \cong K_{1}$ or $K_{2}$, then $P_{5} \sqsubseteq G$, (b) if $\left\langle N(x) \cap N_{0}\right\rangle \cong P_{3}$ or $P_{4}$, then Kite $\sqsubseteq G$, and (c) if $\left\langle N(x) \cap N_{0}\right\rangle \cong K_{1} \cup K_{2}$, then Bull $\sqsubseteq G$, a contradiction. Finally, if $\left\langle N(x) \cap N_{0}\right\rangle \cong 2 K_{1}$ or $C_{5}$, we cannot get $P_{5}$ or $K_{4}$ (or) Kite (or) Bull as an induced subgraph in $\left\langle N_{0} \cup N_{1}\right\rangle$. Hence $\left\langle N(x) \cap N_{0}\right\rangle \cong 2 K_{1}$ or $C_{5}$.

Throughout this proof, for any integer $i, v_{i}$ means $v_{i}(\bmod 5)$ and $A_{i}$ means $A_{i(\bmod 5)}$. For $0 \leq i \leq 4$, let $A_{i}=\left\{x \in N_{1}: N(x) \cap N_{0}=\left\{v_{i-1}, v_{i+1}\right\}\right\} \cup\left\{v_{i}\right\}$ and let $B=\left\{x \in N_{1}\right.$ : $\left.\left\langle N(x) \cap N_{0}\right\rangle \cong C_{5}\right\}$.

Claim 2: $\left\langle\cup_{i=0}^{4} A_{i}\right\rangle \cong \mathbb{I}\left[C_{5}\right]$.

For every $i, 0 \leq i \leq 4$, we have (a) $\left\langle A_{i}\right\rangle$ is independent (else, if $x, y \in A_{i}$ are adjacent, 
then $x, y \neq v_{i}$ and $\left\langle\left\{x, v_{i+1}, y, v_{i-1}, v_{i-2}\right\}\right\rangle \cong$ Kite $\sqsubseteq G$ ), (b) $\left[A_{i}, A_{i+1}\right]$ is complete (else, if $x \in A_{i}$ and $y \in A_{i+1}$ are not adjacent, then $\left.\left\langle\left\{x, v_{i-1}, v_{i-2}, v_{i-3}, y\right\}\right\rangle \cong P_{5} \sqsubseteq G\right)$, (c) $\left[A_{i}, A_{i+2}\right]=\emptyset$ (else, if $x \in A_{i}$ and $y \in A_{i+2}$ are adjacent, then $\left\langle\left\{v_{i-1}, x, v_{i+1}, v_{i+2}, y\right\}\right\rangle \cong$ Bull $\sqsubseteq G)$. Thus from (a), (b) and (c), we conclude that $\left\langle\cup_{i=0}^{4} A_{i}\right\rangle \cong \mathbb{I}\left[C_{5}\right]$.

Claim 3: $\left[\cup_{i=0}^{4} A_{i}, B\right]$ is complete.

On the contrary, if there exist vertices $x \in A_{i}, x \neq v_{i}$ and $y \in B$ such that $x y \notin E(G)$, then $\left\langle\left\{v_{i}, v_{i+1}, x, y, v_{i-2}\right\}\right\rangle \cong$ Bull $\sqsubseteq G$, a contradiction.

Claim 4: $\langle B\rangle$ is independent.

Suppose if there exist vertices $x$ and $y$ in $B$ such that $x y \in E(G)$, then $\left\langle\left\{v_{1}, v_{2}, x, y\right\}\right\rangle \cong$ $K_{4} \sqsubseteq G$, a contradiction.

Claim 5: If $x \in \cup_{i=0}^{4} A_{i}$, then $N(x) \cap N_{2}=\emptyset$.

Let $x \in A_{i}$ for some $i$ such that $0 \leq i \leq 4$. Suppose if there exists a vertex $y \in N(x) \cap N_{2}$, then $\left\langle\left\{y, x, v_{i+1}, v_{i+2}, v_{i+3}\right\}\right\rangle \cong P_{5} \sqsubseteq G$, a contradiction.

Note that, if $B=\emptyset$, then by using claims 2 and 5, we can observe that $G \cong \mathbb{I}\left[C_{5}\right]$. Now, let us assume that $B \neq \emptyset$ and $N_{2} \neq \emptyset$.

Claim 6: $\left[B, N_{2}\right]$ is complete.

Here, if there exist vertices $x \in B$ and $y \in N_{2}$ such that $x y \notin E(G)$, then by using Claim 5, there exists a vertex $z \in B$ such that $y z \in E(G)$. Now from Claim $4, x z \notin E(G)$. Hence $\left\langle\left\{v_{1}, v_{2}, x, y, z\right\}\right\rangle \cong$ Kite $\sqsubseteq G$, a contradiction.

Claim 7: $\left\langle N_{2}\right\rangle$ is triangle-free.

On the contrary, assume that there exist vertices $\left\{u_{1}, u_{2}, u_{3}\right\} \subseteq N_{2}$ which induce a $K_{3}$ in $G$. Then by using Claim 6 , for every vertex $x \in B,\left\langle\left\{x, u_{1}, u_{2}, u_{3}\right\}\right\rangle \cong K_{4} \sqsubseteq G$, a contradiction.

Since $G$ is assumed to be $P_{5}$-free and $\left\langle N_{2}\right\rangle$ is triangle-free, by Theorem 2.2 we see that each component of $\left\langle N_{2}\right\rangle$ is either isomorphic to a $\mathbb{I}\left[C_{5}\right]$ or to a bipartite graph.

Suppose $N_{3}=\emptyset$, by using the above Claims, we see that $G \cong\left\langle\cup_{i=0}^{4} A_{i} \cup N_{2}\right\rangle+\langle B\rangle$. Now, let us assume that $N_{3} \neq \emptyset$.

Claim 8: If $x y$ is an edge in $\left\langle N_{2}\right\rangle$, then $N(x) \cap N_{3}=\emptyset$ and $N(y) \cap N_{3}=\emptyset$.

Let $x y$ be an edge in $\left\langle N_{2}\right\rangle$. Suppose if there exists a vertex $z \in N_{3}$ such that $x z \in E(G)$ or $y z \in E(G)$ (or) $\{x z, y z\} \in E(G)$, then $\left\langle\left\{v_{1}, b, x, y, z\right\}\right\rangle \cong$ Bull or Kite $\sqsubseteq G$ (where $b \in B$ ), a contradiction.

Let $S$ be the collection of the vertices in $N_{2}$ which have neighbors in $N_{3}$. From Claim 8, it can be seen that $S$ is an independent subset of $N_{2}$ such that $\left[S, N_{2} \backslash S\right]=\emptyset$.

Claim 9: There exists a vertex $x^{*} \in S$ such that $\left[x^{*}, N_{3}\right]$ is complete. Also, $\left\langle N_{3}\right\rangle$ is triangle- 
free.

On the contrary, let us assume that there exists no $x^{*} \in S$ such that $\left[x^{*}, N_{3}\right]$ is complete. Under this assumption, first let us show that there exist vertices $x, x^{\prime} \in S$ and $y, y^{\prime} \in N_{3}$ such that $x y, x^{\prime} y^{\prime} \in E(G)$ and $x y^{\prime}, x^{\prime} y \notin E(G)$. Let $x_{1}, x_{2}, \ldots, x_{|S|}$ and $y_{1}, y_{2}, \ldots, y_{\left|N_{3}\right|}$ be the vertices of $S$ and $N_{3}$ respectively. Consider $x_{1}$. By our assumption, $x_{1}$ is non-adjacent to at least one of the vertex in $N_{3}$, say $y_{1}$ and the vertex $y_{1}$ should have a neighbor in $S$, say $x_{2}$. Now the vertex $x_{2}$ is also non-adjacent to at least one vertex in $N_{3}$, say $y_{2}$. Suppose $x_{1} y_{2} \in E(G)$, then $x=x_{1}, x^{\prime}=x_{2}, y=y_{2}$ and $y^{\prime}=y_{1}$ will possess the required property. If not, $x_{1} y_{2} \notin E(G)$ and $y_{2}$ should have a neighbor in $S$, say $x_{3}$. Suppose $x_{3} y_{1} \notin E(G)$, $x=x_{2}, x^{\prime}=x_{3}, y=y_{1}$ and $y^{\prime}=y_{2}$ will have the required property. Otherwise, $x_{3} y_{1} \in E(G)$ and there is a vertex in $N_{3}$ which is non-adjacent to $x_{3}$, say $y_{3}$. Like wise, if $x_{1} y_{3} \in E(G)$ or $x_{2} y_{3} \in E(G)$, then as mentioned above we can get vertices with the required condition. If not, $x_{1} y_{3} \notin E(G)$ and $x_{2} y_{3} \notin E(G)$, and hence the vertex $y_{3}$ should have a neighbor in $S$, say $x_{4}$. Similarly, even when the vertex $x_{4}$ is non-adjacent to $y_{1}$ or $y_{2}$, we can get the vertices with the required condition. Suppose $x_{4}$ is adjacent to $y_{1}$ and $y_{2}$, the process continues. Since the number of vertices is finite, this process stops at a certain stage having vertices $x_{i}, x_{j} \in S$ and $y_{i-1}, y_{i} \in N_{3}$ such that $x_{i} y_{i-1}, y_{i} x_{j} \in E(G)$ and $x_{j} y_{i-1}, x_{i} y_{i} \notin E(G)$ for some $i, j \in\{1,2, \ldots,|S|\}$. Thus there exist vertices $x, x^{\prime} \in S$ and $y, y^{\prime} \in N_{3}$ such that $x y, x^{\prime} y^{\prime} \in E(G)$ and $x y^{\prime}, x^{\prime} y \notin E(G)$. Now by using Claim $8, x x^{\prime} \notin E(G)$, and hence for some $b \in B,\left\langle\left\{v_{1}, b, x^{\prime}, y^{\prime}, y\right\}\right\rangle \cong P_{5}$ when $y y^{\prime} \in E(G)$ or $\left\langle\left\{y, x, b, x^{\prime}, y^{\prime}\right\}\right\rangle \cong P_{5}$ when $y y^{\prime} \notin E(G)$, a contradiction.

Also note that, $\left\langle N_{3}\right\rangle$ has to be triangle-free. Otherwise, $K_{4} \sqsubseteq G$.

Claim 10: $N_{i}=\emptyset$, for all $i \geq 4$.

This can be easily observed from the fact that if $N_{4} \neq \emptyset$, then we will get $P_{5} \sqsubseteq G$, a contradiction.

Since $G$ is $P_{5}$-free and $\left\langle N_{3}\right\rangle$ is triangle-free, each component of $\left\langle N_{3}\right\rangle$ is isomorphic to a $\mathbb{I}\left[C_{5}\right]$ or to a bipartite graph. Let $V_{1}=\left\{\cup_{i=0}^{4} A_{i} \cup\left(N_{2} \backslash S\right)\right\}, V_{2}=B \cup S$ and $V_{3}=N_{3}$. By using the above Claims, we see that $\left\langle V_{2}\right\rangle$ is a complete bipartite graph, $\left\langle V_{1}\right\rangle$ is a disjoint union of $\mathbb{I}\left[C_{5}\right]^{\prime} s$ and bipartite graphs such that $\left[V_{1}, B\right]$ is complete, and $\left\langle V_{3}\right\rangle$ is also a disjoint union of $\mathbb{I}\left[C_{5}\right]^{\prime} s$ and bipartite graphs such that there exists a vertex $x^{*} \in S$ such that $\left[x^{*}, V_{3}\right]$ is complete. Also from Claims 5 and 8, it can be observed that $\left[V_{1}, S\right]=\left[V_{1}, V_{3}\right]=\left[V_{3}, B\right]=\emptyset$.

By Theorem 2.3, one can easily find the chromatic number of this family.

Corollary 2.4 If $G$ is a connected $\left\{P_{5}, K_{4}, K\right.$ ite, Bull $\}$-free graph that contains an induced 
$C_{5}$, then $\chi(G)=3$ if and only if $G \cong \mathbb{I}\left[C_{5}\right]$, otherwise $\chi(G)=4$.

Proof. By Theorem 2.3, we see that $V(G)=V_{1} \cup V_{2} \cup V_{3}$, where $V_{1}, V_{2}$ and $V_{3}$ have the properties stated in the statement of Theorem 2.3. Since $\left\langle V_{1} \cup V_{3}\right\rangle$ is a disjoint union of $\mathbb{I}\left[C_{5}\right]^{\prime} s$ and bipartite graphs, one can color the vertices of $V_{1}$ and $V_{3}$ with colors $\{1,2,3\}$ and $\{2,3,4\}$ respectively which yields a proper coloring for the subgraph $\left\langle V_{1} \cup V_{3}\right\rangle$. Since $\left\langle V_{2}\right\rangle$ is a complete bipartite graph with bipartition $B$ and $S,\left[B, V_{1}\right]$ is complete, $\left[x^{*}, V_{3}\right]$ is complete and $\left[V_{1}, S\right]=\left[V_{1}, V_{3}\right]=\left[V_{3}, B\right]=\emptyset$, coloring the vertices of $B$ and $S$ with 4 and 1 respectively will yields a proper coloring for $G$. Thus $\chi(G) \leq 4$. Suppose $B=\emptyset$, then $G \cong \mathbb{I}\left[C_{5}\right]$ and hence $\chi(G)=3$. If not, $B \neq \emptyset$ and $\langle B\rangle+\mathbb{I}\left[C_{5}\right] \sqsubseteq G$. Thus $\chi(G)=4$.

Now we shall consider the indicated coloring for the independent expansion of $C_{n}$.

Theorem 2.5 For $1 \leq i \leq n$, let $m_{i}$ 's be positive integers. Then the graph $G=$ $\mathbb{I}\left[C_{n}\right]\left(m_{1}, m_{2}, \ldots, m_{n}\right)$ is $k$-indicated colorable for all $k \geq \chi(G)$.

Proof. For the graph $G=\mathbb{I}\left[C_{n}\right]\left(m_{1}, m_{2}, \ldots, m_{n}\right)$, for $m_{i} \geq 1,1 \leq i \leq n$, it is easy to observe that $\chi(G)=2$ when $n$ is even and $\chi(G)=3$ when $n$ is odd. Here, if Ann first present the vertices of an induced $C_{n}$ cyclically and then the remaining vertices in any order, Ben will not be able to produce a blocked vertex. Thus Ann has a winning strategy for $G$ with $k$ colors, for every $k \geq \chi(G)$.

We know that, for the union of two graphs $G_{1}$ and $G_{2}, \chi\left(G_{1} \cup G_{2}\right)=\max \left\{\chi\left(G_{1}\right), \chi\left(G_{2}\right)\right\}$. The same holds even for the indicated chromatic number.

Theorem 2.6 (13]). Let $G=G_{1} \cup G_{2}$. If $G_{1}$ is $k_{1}$-indicated colorable for every $k_{1} \geq \chi_{i}\left(G_{1}\right)$ and $G_{2}$ is $k_{2}$-indicated colorable for every $k_{2} \geq \chi_{i}\left(G_{2}\right)$, then $\chi_{i}(G)=\max \left\{\chi_{i}\left(G_{1}\right), \chi_{i}\left(G_{2}\right)\right\}$ and $G$ is $k$-indicated colorable for all $k \geq \chi_{i}(G)$.

Next, we see that Corollary 2.7 which was proved in [13] is a simple consequence of Theorem 2.1, 2.2, 2.5 and 2.6.

Corollary 2.7 ([13]) Every $\left\{P_{5}, K_{3}\right\}$-free graph $G$ is $k$-indicated colorable for all $k \geq \chi(G)$.

Now, let us consider the indicated coloring of $\left\{P_{5}, K_{4}, K i t e, B u l l\right\}$-free graphs which contains an induced $C_{5}$. 
Theorem 2.8 Let $G$ be a $\left\{P_{5}, K_{4}, K i t e, B u l l\right\}$-free graph which contains an induced $C_{5}$. Then $G$ is $k$-indicated colorable for all $k \geq \chi(G)$.

Proof. By Theorem 2.6, it is enough to prove the result for a connected $\left\{P_{5}, K_{4}\right.$, Kite, Bull $\}$ free graph that contains an induced $C_{5}$. Let $G$ be such a graph. Then by Theorem $2.3, V(G)=$ $V_{1} \cup V_{2} \cup V_{3}$ where (1) $\left\langle V_{2}\right\rangle$ is a complete bipartite graph with bipartition say $B$ and $S$, (2) $\left\langle V_{1} \cup V_{3}\right\rangle$ is a disjoint union of $\mathbb{I}\left[C_{5}\right]^{\prime} s$ and bipartite graphs, (3) $\left[V_{1}, B\right]$ is complete, $\left[V_{1}, S\right]=\emptyset$, $\left[V_{1}, V_{3}\right]=\emptyset,\left[V_{3}, B\right]=\emptyset$ and (4) there exists a vertex, say $x^{*} \in S$ such that $\left[x^{*}, V_{3}\right]$ is complete.

Suppose $B=\emptyset$, then $G \cong \mathbb{I}\left[C_{5}\right]$. Thus by Theorem 2.5, $G$ is $k$-indicated colorable for all $k \geq \chi(G)$. If not, $B \neq \emptyset$ and hence by Corollary 2.4, $G ¥ \mathbb{I}\left[C_{5}\right]$ and $\chi(G)=4$. Let $\{1,2, \ldots, k \geq 4\}$ be the set of colors. We shall show that $G$ is $k$-indicated colorable. Let Ann start by presenting $x^{*}$ and a vertex $b \in B$. Without loss of generality, let the color used by Ben for $b$ and $x^{*}$ be 1 and 2 respectively. Since $\left[b, V_{1}\right]$ is complete and $\left[x^{*}, V_{3}\right]$ is complete, the set of available colors for $V_{1}$ and $V_{2}$ are $\{2,3, \ldots, k\}$ and $\{1,3,4, \ldots, k\}$ respectively. Since $\left[V_{1}, V_{3}\right]=\emptyset,\left\langle V_{1} \cup V_{3}\right\rangle$ is a disjoint union of $\mathbb{I}\left[C_{5}\right]^{\prime} s$ and bipartite graphs, by Theorem 2.1 and Theorem 2.5, $\left\langle V_{1} \cup V_{3}\right\rangle$ is $l$-indicated colorable for all $l \geq 3$. That is, Ann has a winning strategy for $\left\langle V_{1}\right\rangle$ while using the colors $\{2,3, \ldots, k\}$ and a winning strategy for $\left\langle V_{3}\right\rangle$ while using the colors $\{1,3,4, \ldots, k\}$. After presenting the vertices of $V_{1}$ and $V_{3}$ by using these winning strategies, Ann will present the remaining vertices of $B$ and $S$ in any order. Clearly, the color of the vertices $b$ and $x^{*}$, namely 1 and 2 are available for the uncolored vertices of $B$ and $S$ respectively. Thus Ann wins the game on $G$ with $k$ colors, $k \geq 4$.

Next, let us consider a structural characterization of a family of $P_{6}$-free graphs. The study of $P_{6}$-free graphs has also been of interest for a lot of coloring parameters. See for instance, [10, 12. 14]. Here, we would like to consider connected $\left\{P_{6}, C_{5}, K_{1,3}\right\}$-free graphs that contains an induced $C_{6}$.

Theorem 2.9 If $G$ is a connected $\left\{P_{6}, C_{5}, K_{1,3}\right\}$-free graph which contains an induced $C_{6}$ then $G$ is isomorphic to the graph given in Figure 2. Here $V(G)=\left(\cup_{i=0}^{5} A_{i}\right) \cup\left(\cup_{j=0}^{2} B_{j}\right)$ and the circle denote the complete subgraph induced by the sets $A_{i}$ and $B_{j}$ and the double line between any two sets denote the join of the two sets.

Proof. Let $G$ be a connected $\left\{P_{6}, C_{5}, K_{1,3}\right\}$-free graph that contains an induced $C_{6} \cong$ $\left\langle\left\{v_{0}, v_{1}, v_{2}, v_{3}, v_{4}, v_{5}\right\}\right\rangle=\left\langle N_{0}\right\rangle$, and let $N_{i}=\left\{x \in V(G): \operatorname{dist}\left(x, N_{0}\right)=i\right\}, i \geq 1$.

Claim 1: If $x \in N_{1}$, then $\left\langle N(x) \cap N_{0}\right\rangle \cong P_{3}$ or $2 K_{2}$.

For $x \in N_{1}$, the possibilities for $\left\langle N(x) \cap N_{0}\right\rangle$ are $K_{1}, K_{2}, P_{3}, P_{4}, P_{5}, 2 K_{1}, 3 K_{1}, 2 K_{2}, K_{1} \cup$ 


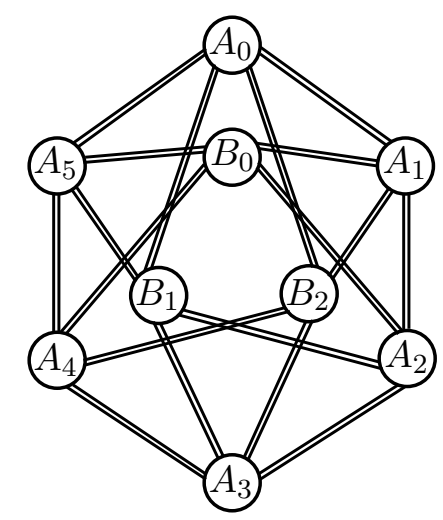

Figure 2: $\left\{P_{6}, C_{5}, K_{1,3}\right\}$-free graph contains an induced $C_{6}$

$K_{2}, K_{1} \cup P_{3}$ and $C_{6}$. Here (a) if $\left\langle N(x) \cap N_{0}\right\rangle \cong K_{1}$ or $K_{2}$, then $P_{6} \sqsubseteq G$, (b) if $\left\langle N(x) \cap N_{0}\right\rangle \cong$ $P_{4}$ or $K_{1} \cup K_{2}$, then $C_{5} \sqsubseteq G$, (c) if $\left\langle N(x) \cap N_{0}\right\rangle \cong P_{5}$ or $C_{6}$ (or) $K_{1} \cup P_{3}$ (or) $3 K_{1}$ (or) $2 K_{1}$, then $K_{1,3} \sqsubseteq G$, a contradiction. Finally, if $\left\langle N(x) \cap N_{0}\right\rangle \cong P_{3}$ or $2 K_{2}$, we see that neither $P_{6}$ nor $C_{5}$ (nor) $K_{1,3}$ is an induced subgraph of $\left\langle N_{0} \cup N_{1}\right\rangle$. Thus $\left\langle N(x) \cap N_{0}\right\rangle \cong P_{3}$ or $2 K_{2}$.

Throughout this proof, for any integer $i, v_{i}$ means $v_{i}(\bmod 6)$ and $A_{i}$ means $A_{i}(\bmod 6)$. For $0 \leq i \leq 5$, let $A_{i}=\left\{x \in N_{1}: N(x) \cap N_{0}=\left\{v_{i-1}, v_{i}, v_{i+1}\right\}\right\} \cup\left\{v_{i}\right\}$ and $B_{i}=\left\{x \in N_{1}\right.$ : $\left.N(x) \cap N_{0}=\left\{v_{i-2}, v_{i-1}, v_{i+1}, v_{i+2}\right\}\right\}$.

Claim 2: $\left\langle\cup_{i=0}^{5} A_{i}\right\rangle \cong \mathbb{K}\left[C_{6}\right]$.

For every $i, 0 \leq i \leq 5$, we have (a) $\left\langle A_{i}\right\rangle$ is complete (suppose if there exist vertices $x, y \in A_{i}$ such that $x y \notin E(G)$, then $\left.\left\langle\left\{v_{i+1}, v_{i+2}, x, y\right\}\right\rangle \cong K_{1,3} \sqsubseteq G\right)$, (b) $\left[A_{i}, A_{i+1}\right]$ is complete, (if not, there exist vertices $x \in A_{i}$ and $y \in A_{i+1}$ such that $x y \notin E(G)$, and hence $\left\langle\left\{x, v_{i}, y, v_{i+2}, v_{i+3}, v_{i+4}\right\}\right\rangle \cong P_{6} \sqsubseteq G$ ), (c) $\left[A_{i}, A_{i+2}\right]=\emptyset$, (suppose if there exist vertices $x \in A_{i}$ and $y \in A_{i+2}$ such that $x y \in E(G)$, then $\left.\left\langle\left\{x, y, v_{i+3}, v_{i+4}, v_{i+5}\right\}\right\rangle \cong C_{5} \sqsubseteq G\right),(\mathrm{d})$ $\left[A_{i}, A_{i+3}\right]=\emptyset$, (otherwise as shown previously, we can find $x \in A_{i}$ and $y \in A_{i+3}$ such that $x y \in E(G)$, and $\left.\left\langle\left\{x, v_{i-1}, v_{i+1}, y\right\}\right\rangle \cong K_{1,3} \sqsubseteq G\right)$. Thus from (a), (b), (c) and (d), it can be seen that $\left\langle\cup_{i=0}^{5} A_{i}\right\rangle \cong \mathbb{K}\left[C_{6}\right]$.

Claim 3: $\left\langle B_{i}\right\rangle$ is complete, for $i=0,1,2,3,4,5$.

Here, if there exist vertices $x, y \in B_{i}$ such that $x y \notin E(G)$, then $\left\langle\left\{v_{i-1}, v_{i}, x, y\right\}\right\rangle \cong$ $K_{1,3} \sqsubseteq G$, a contradiction.

Claim 4: $\left[B_{i}, B_{i+1}\right]=\emptyset$, for $i=0,1,2,3,4,5$.

Suppose if there exist vertices $x \in B_{i}$ and $y \in B_{i+1}$ such that $x y \in E(G)$, then $\left\langle\left\{x, y, v_{i+1}, v_{i-2}\right\}\right\rangle \cong$ $K_{1,3} \sqsubseteq G$, a contradiction.

Claim 5: $\left[A_{i}, B_{i}\right]=\emptyset, i=0,1,2,3,4,5$.

On the contrary, if there exist vertices $x \in A_{i}$ and $y \in B_{i}$ such that $x y \in E(G)$, then 
$\left\langle\left\{y, x, v_{i-2}, v_{i+2}\right\}\right\rangle \cong K_{1,3} \sqsubseteq G$, a contradiction.

Claim 6: $\left[A_{i}, B_{i+1}\right]$ is complete, for $i=0,1,2,3,4,5$.

If not, there exist vertices $x \in A_{i}$ and $y \in B_{i+1}$ such that $x y \notin E(G)$. Here $\left\langle\left\{x, v_{i-1}, y, v_{i+2}, v_{i+1}\right\}\right\rangle \cong$ $C_{5} \sqsubseteq G$, a contradiction.

Claim 7: $\left[A_{i}, B_{i+2}\right]$ is complete, for $i=0,1,2,3,4,5$.

It is easy to observe that if there exist vertices $x \in A_{i}$ and $y \in B_{i+2}$ such that $x y \notin E(G)$, then $\left\langle\left\{x, v_{i-1}, v_{i-2}, y, v_{i+1}\right\}\right\rangle \cong C_{5} \sqsubseteq G$, a contradiction.

Claim 8: $N_{i}=\emptyset$, for all $i, i \geq 2$.

It is enough to show that $N_{2}=\emptyset$. Suppose $N_{2} \neq \emptyset$, then there exists a vertex $x \in N_{2}$. Since $G$ is connected, there exists a vertex $y \in A_{j}$ or $y \in B_{j}$ for some $j \in\{0,1, \ldots, 5\}$ such that $x y \in E(G)$. Then $\left\langle\left\{y, v_{j-1}, v_{j+1}, x\right\}\right\rangle \cong K_{1,3} \sqsubseteq G$, a contradiction. Thus $V(G)=N_{0} \cup N_{1}$.

Note that $B_{j}=B_{j+3}$ for every $j \in\{0,1,2\}$. From all these Claims, we see that $G$ will be isomorphic to the graph shown in Figure 2 .

An immediate consequence of Theorem 2.9 is given in Corollary 2.10.

Corollary 2.10 If $G$ is a connected $\left\{P_{6}, C_{5}, \overline{P_{5}}, K_{1,3}\right\}$-free graph that contains an induced $C_{6}$ then $G \cong \mathbb{K}\left[C_{6}\right]$.

Proof. It can seen from Theorem 2.9 that it is enough to show that $B_{i}=\left\{x \in N_{1}: N(x) \cap\right.$ $\left.N_{0}=\left\{v_{i-2}, v_{i-1}, v_{i+1}, v_{i+2}\right\}\right\}=\emptyset$, for every $i=0,1,2$. Suppose $x \in B_{i}$ for some $i \in$ $\{0,1,2\}$, then $\left\langle\left\{x, v_{i-1}, v_{i}, v_{i+1}, v_{i+2}\right\}\right\rangle \cong \overline{P_{5}} \sqsubseteq G$, a contradiction. Thus $G$ is isomorphic to a complete expansion of $C_{6}$.

Even though the graph $G$ shown in Figure 2 looks simple, it looks challenging to obtain the indicated chromatic number of $G$. So, we have considered the indicated coloring of $\mathbb{K}\left[C_{6}\right]$.

Proposition 2.11 For $1 \leq i \leq 6$, let $m_{i}$ 's be positive integers. Then the graph $G=$ $\mathbb{K}\left[C_{6}\right]\left(m_{1}, m_{2}, m_{3}, m_{4}, m_{5}, m_{6}\right)$ is $k$-indicated colorable for all $k \geq \chi(G)$.

Proof. Let us consider the graph $G=\mathbb{K}\left[C_{6}\right]\left(m_{1}, m_{2}, m_{3}, m_{4}, m_{5}, m_{6}\right)$, where $m_{i} \geq 1$ and $V_{i}=V\left(K_{m_{i}}\right)$ for $1 \leq i \leq 6$. Let $k$ be a positive integer such that $k \geq \chi(G)$ and let $\{1,2, \ldots, k\}$ be the set of colors. We shall show that $G$ is $k$-indicated colorable. It is easy to see that $\chi(G)=\omega(G)$. Let Ann start by presenting the vertices of a maximum clique. Without loss of generality, let it be $V_{1} \cup V_{2}$. Since $k \geq \omega(G)$, Ben has an available color for all the vertices in $V_{1} \cup V_{2}$ and let the colors given to $V_{1}$ and $V_{2}$ be $\left\{1,2, \ldots, m_{1}\right\}$ and 
$\left\{m_{1}+1, m_{1}+2, \ldots, m_{1}+m_{2}=\omega(G)\right\}$ respectively. Now, let Ann present the vertices of $V_{3}$ and $V_{6}$ (in any order). Since $V_{1} \cup V_{2}$ is maximum clique, $\left|V_{3}\right| \leq\left|V_{1}\right|$ and $\left|V_{6}\right| \leq\left|V_{2}\right|$, and hence Ben has an available colors for all the vertices of $V_{3}$ and $V_{6}$. Since $\left[V_{i}, V_{i+1}\right]$ is complete, $\left\{1,2, \ldots, m_{1}\right\}$ and $\left\{m_{1}+1, m_{1}+2, \ldots, \omega(G)\right\}$ are colors available for $V_{5}$ and $V_{4}$ respectively. Now, let Ann present the vertices of $V_{4}$ until either the number of available colors for $V_{5}$ is equal to $\left|V_{5}\right|$ or every vertex in $V_{4}$ is colored. In either case, let Ann proceed by presenting all the vertices of $V_{5}$. If there are some uncolored vertices in $V_{4}$, let Ann present those vertices finally. Here it can be seen that, the number of available color for the vertices of $V_{4} \cup V_{5}$ is at least $\omega(G)$ and $\left|V_{4}\right|+\left|V_{5}\right| \leq \omega(G)$. Thus in this ordering, Ben will always have an available color for the remaining vertices.

An immediate consequence of Theorem 2.6. Corollary 2.10 and Proposition 2.11 is given in Corollary 2.12.

Corollary 2.12 If $G$ is a $\left\{P_{6}, C_{5}, \overline{P_{5}}, K_{1,3}\right\}$-free graph that contains an induced $C_{6}$, then $G$ is $k$-indicated colorable for all $k \geq \chi(G)$.

\section{Indicated coloring of $\mathbb{K}\left[C_{5}\right]$ and some of its consequences}

Let us start this Section by recalling two of the results which were proved in [13].

Theorem 3.1 ([13]). Any graph $G$ is $k$-indicated colorable for all $k \geq \operatorname{col}(G)$.

We know that, for the join of two graphs $G_{1}$ and $G_{2}, \chi\left(G_{1}+G_{2}\right)=\chi\left(G_{1}\right)+\chi\left(G_{2}\right)$. The same holds even for the indicated chromatic number.

Theorem 3.2 ([13]). Let $G=G_{1}+G_{2}$. If $G_{1}$ is $k_{1}$-indicated colorable for every $k_{1} \geq \chi_{i}\left(G_{1}\right)$ and $G_{2}$ is $k_{2}$-indicated colorable for every $k_{2} \geq \chi_{i}\left(G_{2}\right)$, then $\chi_{i}(G)=\chi_{i}\left(G_{1}\right)+\chi_{i}\left(G_{2}\right)$ and $G$ is $k$-indicated colorable for all $k \geq \chi_{i}(G)$.

Let us recall the structural characterization of $\left\{P_{2} \cup P_{3}, C_{4}\right\}$-free graphs, $\left\{P_{5}, C_{4}\right\}$-free graphs and $\left\{P_{5},\left(\overline{P_{2} \cup P_{3}}\right), \overline{P_{5}}\right.$, Dart $\}$-free graphs which contains an induced $C_{5}$. The graphs $\left(\overline{P_{2} \cup P_{3}}\right)$ and Dart are shown in Figure1.

Theorem 3.3 ([5]) If $G$ is a connected $\left\{P_{2} \cup P_{3}, C_{4}\right\}$-free graph, then $G$ is chordal or there exists a partition $\left(V_{1}, V_{2}, V_{3}\right)$ of $V(G)$ such that $(1)\left\langle V_{1}\right\rangle \cong \overline{K_{m}}$, for some $m \geq 0$, (2) $\left\langle V_{2}\right\rangle \cong$ 

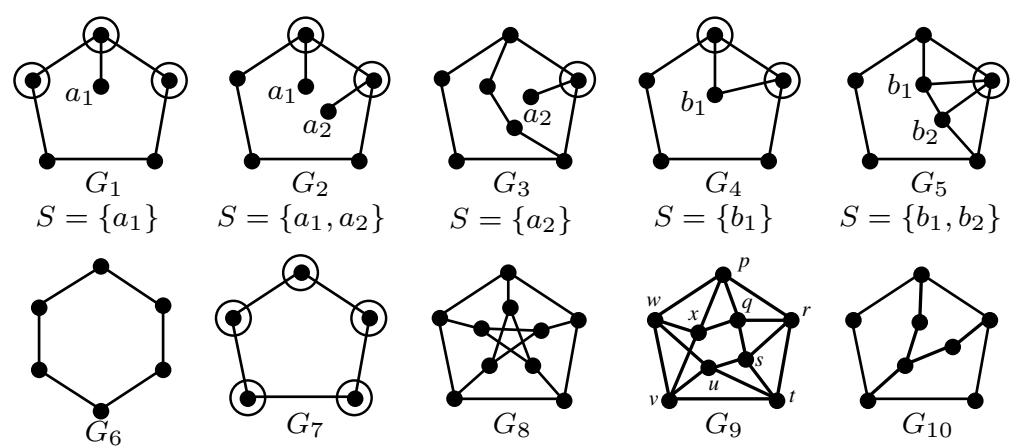

$S=\left\{b_{1}, b_{2}\right\}$
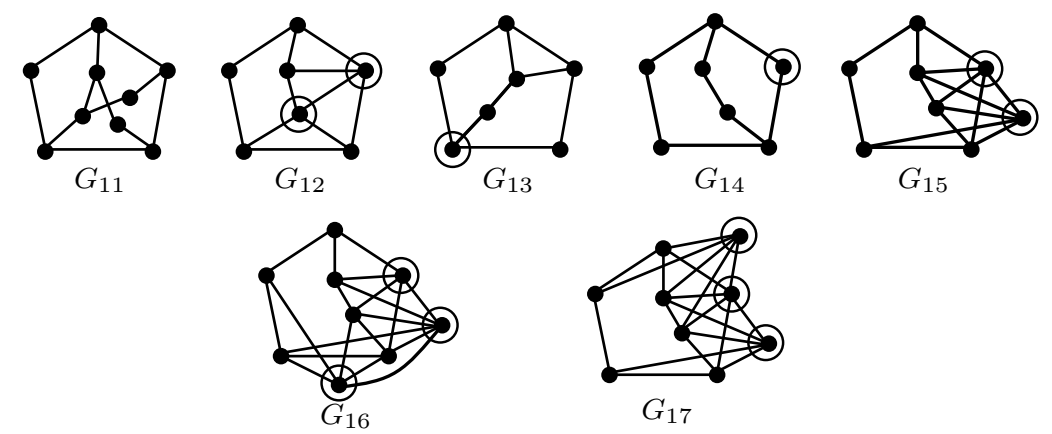

Figure 3: Basic graphs used in Theorem $3.3\left(S=\emptyset\right.$ for $\left.G_{i}, 6 \leq i \leq 17\right)$

$K_{t}$, for some $t \geq 0$, (3) $\left\langle V_{3}\right\rangle$ is isomorphic to a graph obtained from one of the basic graphs $G_{t}(1 \leq t \leq 17)$ shown in Figure 3 by expanding each vertex indicated in circle by a complete graph (of order $\geq 1$ ), (4) $\left[V_{1}, V_{3}\right]=\emptyset$ and (5) $\left[V_{2}, V_{3} \backslash S\right]$ is complete.

Theorem 3.4 ([6]) Let $G$ be a connected $\left\{P_{5}, C_{4}\right\}$-free graph. Then $V(G)=V_{1} \cup V_{2}$ such that (i) $\left\langle V_{1}\right\rangle$ is a $P_{5}$-free graph which is also chordal.

(ii) If $V_{2} \neq \emptyset$, then $\left\langle V_{2}\right\rangle=A_{1} \cup A_{2} \cup \cdots \cup A_{l}$ where each $A_{i}$ is a $\mathbb{K}\left[C_{5}\right]$, for every $i \in$ $\{1,2, \ldots, l\}$ for some $l \geq 1$. Also, $\left\langle N\left(A_{i}\right)\right\rangle$ is a complete subgraph of $V_{1}$ and $\left[A_{i}, N\left(A_{i}\right)\right]$ is complete.

Theorem 3.5 ([1] $]$ If $G$ is a connected $\left\{P_{5},\left(\overline{P_{2} \cup P_{3}}\right), \overline{P_{5}}\right.$, Dart $\}$-free graph that contains an induced $C_{5}$, then $G$ is either isomorphic to $C_{5}\left(S_{1}, S_{2}, S_{3}, S_{4}, S_{5}\right)$ or $C_{5}\left(S_{1}, S_{2}, S_{3}, S_{4}, S_{5}\right)+H$, where $S_{i}^{\prime} s$ are induced split subgraphs of $G, H$ is nonempty and $H \sqsubseteq G$.

We further claim that the subgraph $H$ mentioned in Theorem 3.5 is complete. Let $G$ be a connected $\left\{P_{5},\left(\overline{P_{2} \cup P_{3}}\right), \overline{P_{5}}\right.$, Dart $\}$-free graph that contains an induced $C_{5}$. Let $C=$ $\left\langle\left\{v_{0}, v_{1}, v_{2}, v_{3}, v_{4}\right\}\right\rangle \cong C_{5} \sqsubseteq G$. Suppose there exists two non-adjacent vertices $x$ and $y$ in $H$. Then $\left\langle\left\{v_{1}, v_{2}, x, y, v_{4}\right\}\right\rangle \cong\left(\overline{P_{2} \cup P_{3}}\right) \sqsubseteq G$, a contradiction. 
It can be noted that $\mathbb{K}\left[C_{5}\right]$ is one of the graphs mentioned in Figure 3 of Theorem 3.3 . Also $\mathbb{K}\left[C_{5}\right]$ is an induced subgraph of the graphs mentioned in Theorem 3.4 and 3.5 . So, we shall first consider the indicated coloring for the complete expansion of $C_{5}$. Even though $\mathbb{K}\left[C_{5}\right]$ looks simple, a technique as done for $\mathbb{K}\left[C_{6}\right]$ (see Proposition 2.11) doesn't look possible for $\mathbb{K}\left[C_{5}\right]$. Hence we are forced to adopt a laborious process.

Theorem 3.6 For $1 \leq i \leq 5$, let $m_{i}$ 's be positive integers. Then the graph $G=$ $\mathbb{K}\left[C_{5}\right]\left(m_{1}, m_{2}, m_{3}, m_{4}, m_{5}\right)$ is $k$-indicated colorable for all $k \geq \chi(G)$.

Proof. Let the graph $G=\mathbb{K}\left[C_{5}\right]\left(m_{1}, m_{2}, m_{3}, m_{4}, m_{5}\right)$, where $m_{i} \geq 1$ and $V_{i}=V\left(K_{m_{i}}\right)$ for $1 \leq i \leq 5$. Also, let $k$ be a positive integer such that $k \geq \chi(G)$ and let $\{1,2, \ldots, k\}$ be the set of colors. We shall show that $G$ is $k$-indicated colorable. Without much difficult, it can be seen that $\alpha(G)=2$. Suppose $\omega(G) \geq \frac{|V(G)|}{\alpha(G)}=\frac{|V(G)|}{2}$, let Ann start by presenting the vertices of a maximum clique. Without loss of generality, let it be $V_{1} \cup V_{2}$. By our choice of $k, k \geq \chi(G)$, and hence $|V(G)| \leq 2 \omega(G) \leq 2 k$. Next, let Ann present the vertices of $V_{3}$ and $V_{5}$ in any order. Since $V_{1} \cup V_{5}$ and $V_{2} \cup V_{3}$ induce a clique and $V_{1} \cup V_{2}$ is a maximum clique, we see that $\left|V_{5}\right| \leq\left|V_{2}\right|$ and $\left|V_{3}\right| \leq\left|V_{1}\right|$. Thus Ben will have an available color for each of the vertex in $V_{3}$ and $V_{5}$. Finally, let Ann present the vertices of $V_{4}$. As we have already observed, $|V(G)| \leq 2 \omega(G)$ and hence $\left|V_{1}\right|+\ldots+\left|V_{5}\right| \leq 2\left(\left|V_{1}\right|+\left|V_{2}\right|\right)$ which in turn implies that $\left|V_{3}\right|+\left|V_{4}\right|+\left|V_{5}\right| \leq k$. Thus here also Ben has an available color for the vertices of $V_{4}$. Hence, Ann wins the game on $k$ colors.

Now let us consider the case when $\omega(G)<\frac{|V(G)|}{2}$. We know that, $\frac{|V(G)|}{2} \leq \chi(G) \leq k$. In this case, let Ann first present the vertices of $V_{1}$. For $2 \leq i \leq 5$, let $\mathcal{N}_{i}$ denote the set of all uncolored vertices in $V_{i}$ and $\mathcal{C}_{i}$ denote the set of available colors for the vertices in $\mathcal{N}_{i}$. Let $c(v)$ denote the color given by Ben to the vertex $v$.

The following are some of the observation regarding $\mathcal{N}_{i}$ and $\mathcal{C}_{i}$.

\section{Observations 3.6.1}

(i) Once when the vertices of $V_{1}$ are colored by Ben, we have the following values.

$$
\begin{gathered}
\left|\mathcal{C}_{i}\right|-\left|\mathcal{N}_{i}\right|=k-\left|V_{1}\right|-\left|V_{i}\right|>0, \text { for } i \in\{2,5\} \\
\left|\mathcal{C}_{i}\right|-\left|\mathcal{N}_{i}\right|=k-\left|V_{i}\right|>0, \text { for } i \in\{3,4\} \\
\left|\mathcal{C}_{i} \cup \mathcal{C}_{i+1}\right|-\left|\mathcal{N}_{i}\right|-\left|\mathcal{N}_{i+1}\right|=k-\left(\left|V_{i}\right|+\left|V_{i+1}\right|\right)>0, \text { for } i \in\{2,3,4\}
\end{gathered}
$$

(Note that, since $k>\omega(G)$, all the values given in the above equations are positive. Also note that, we shall use \{\}$^{*}$ to indicate these values. For instance, $\left.\left\{\left|\mathcal{C}_{2}\right|-\left|\mathcal{N}_{2}\right|\right\}^{*}=k-\left|V_{1}\right|-\left|V_{2}\right|\right)$. 
(ii) For $2 \leq i \leq 5$, the sets $\mathcal{N}_{i}$ and $\mathcal{C}_{i}$ constantly change during the coloring process.

(iii) For $2 \leq i \leq 5$, if $\left|\mathcal{C}_{i}\right|-\left|\mathcal{N}_{i}\right| \geq 0$, then Ben has an available color at that stage for each of the vertex in $V_{i}$.

(iv) On coloring the vertices of $V_{i}$, the value of $\left|\mathcal{C}_{i}\right|-\left|\mathcal{N}_{i}\right|$ remains unchanged (since both $\left|\mathcal{C}_{i}\right|$ and $\left|\mathcal{N}_{i}\right|$ are reduced by 1$)$.

(v) Similarly, on coloring the vertices of $V_{i} \cup V_{i+1}$, the value of $\left|\mathcal{C}_{i} \cup \mathcal{C}_{i+1}\right|-\left|\mathcal{N}_{i}\right|-\left|\mathcal{N}_{i+1}\right|$ remains unchanged.

Let us now proceed with the other uncolored vertices, namely, $V_{2} \cup V_{3} \cup V_{4} \cup V_{5}$. Without loss of generality, let us assume that $\left|V_{3}\right| \geq\left|V_{4}\right|$ and hence $\left|\mathcal{C}_{3}\right|-\left|\mathcal{N}_{3}\right| \leq\left|\mathcal{C}_{4}\right|-\left|\mathcal{N}_{4}\right|$. Now let Ann present the vertices of $V_{3}$ until one of the following holds.

(i). All the vertices of $V_{3}$ are colored

(ii). $\left|\mathcal{C}_{2}\right|-\left|\mathcal{N}_{2}\right|=0$

(iii). $\left|\mathcal{C}_{4} \cup \mathcal{C}_{5}\right|-\left|\mathcal{N}_{4}\right|-\left|\mathcal{N}_{5}\right|=0$.

Case 1 : (i) holds.

In this case, we can easily observe that $\left|\mathcal{C}_{4} \cup \mathcal{C}_{5}\right|-\left|\mathcal{N}_{4}\right|-\left|\mathcal{N}_{5}\right| \geq 0$ and $\left|\mathcal{C}_{i}\right|-\left|\mathcal{N}_{i}\right| \geq 0$, $i=2,4,5$. Next, let Ann present the vertices of $V_{2}$ in any order. Since $\left|\mathcal{C}_{2}\right|-\left|\mathcal{N}_{2}\right| \geq 0$, Ben always has an available color for the vertices of $V_{2}$. Now for presenting the vertices of $V_{4} \cup V_{5}$, let Ann follow the following strategy.

Compare $\left|\mathcal{C}_{4}\right|-\left|\mathcal{N}_{4}\right|$ and $\left|\mathcal{C}_{5}\right|-\left|\mathcal{N}_{5}\right|$. Which ever is smaller Ann present an uncolored vertex from that vertex set, namely, $V_{4}$ or $V_{5}$. Do this again and again until $\mathcal{N}_{4} \cup \mathcal{N}_{5}=\emptyset$.

Note that even after presenting the vertices of $V_{2},\left|\mathcal{C}_{i}\right|-\left|\mathcal{N}_{i}\right| \geq 0$ for $i=4$ and 5 , and $\left|\mathcal{C}_{4} \cup \mathcal{C}_{5}\right|-\left|\mathcal{N}_{4} \cup \mathcal{C}_{5}\right| \geq 0$. This together with (iv) and (v) of Observation 3.6.1 guarantees that when Ann follows the above strategy for presenting the uncolored vertices in $V_{4} \cup V_{5}$, Ben will have an available color for each of these vertices.

Case 2 : (ii) holds.

For $\left|\mathcal{C}_{2}\right|-\left|\mathcal{N}_{2}\right|=0$, Ben should have colored the vertices of $V_{3}$ with exactly $\left\{\left|\mathcal{C}_{2}\right|-\right.$ $\left.\left|\mathcal{N}_{2}\right|\right\}^{*}=k-\left|V_{1}\right|-\left|V_{2}\right|$ colors which are not given to the vertices of $V_{1}$. Next, let Ann present the vertices of $V_{2}$. Since $\left|\mathcal{C}_{2}\right|-\left|\mathcal{N}_{2}\right|=0$, by using (iii) of Observation 3.6.1, Ben has an available color for each of the vertex in $V_{2}$. One can easily observe that every time a vertex is colored in $V_{2}$, the value of $\left|\mathcal{C}_{3}\right|-\left|\mathcal{N}_{3}\right|$ is reduced by 1 . Also $\left|V_{2}\right|+\left|V_{3}\right| \leq \omega(G)<k$ and none of the vertex in $V_{4}$ is colored. Hence $\left|\mathcal{C}_{3}\right|-\left|\mathcal{N}_{3}\right| \geq 0$. Now, let Ann present all the uncolored vertices of $V_{3}$. Again by (iii) of Observation 3.6.1, Ben has an available color for each of the vertex presented. A similar argument shows that $\left|\mathcal{C}_{4}\right|-\left|\mathcal{N}_{4}\right| \geq 0$ and $\left|\mathcal{C}_{5}\right|-\left|\mathcal{N}_{5}\right| \geq 0$. As observed earlier, Ben must have colored the vertices of $V_{3}$ with exactly 
$\left\{\left|\mathcal{C}_{2}\right|-\left|\mathcal{N}_{2}\right|\right\}^{*}=k-\left|V_{1}\right|-\left|V_{2}\right|$ colors which are not given to the vertices of $V_{1}$. Hence, we see that $\left|\left\{c(v): v \in V_{3}\right\} \cap\left\{c(v): v \in V_{1}\right\}\right|=\left|V_{3}\right|-\left\{\left|\mathcal{C}_{2}\right|-\left|\mathcal{N}_{2}\right|\right\}^{*}=\left|V_{3}\right|-\left(k-\left|V_{1}\right|-\left|V_{2}\right|\right)$. Also observe that, the value of $\left|\mathcal{C}_{4} \cup \mathcal{C}_{5}\right|-\left|\mathcal{N}_{4}\right|-\left|\mathcal{N}_{5}\right|$ reduces by one every time Ben colors a vertex of $V_{3}$ with a color given to a vertex in $V_{1}$. Now,

$$
\begin{aligned}
\left|\mathcal{C}_{4} \cup \mathcal{C}_{5}\right|-\left|\mathcal{N}_{4}\right|-\left|\mathcal{N}_{5}\right| & =\left\{\left|\mathcal{C}_{4} \cup \mathcal{C}_{5}\right|-\left|\mathcal{N}_{4}\right|-\left|\mathcal{N}_{5}\right|\right\}^{*}-\left|\left\{c(v): v \in V_{3}\right\} \cap\left\{c(v): v \in V_{1}\right\}\right| \\
& =\left(k-\left|V_{4}\right|-\left|V_{5}\right|\right)-\left(\left|V_{3}\right|-\left(k-\left|V_{1}\right|-\left|V_{2}\right|\right)\right) \\
& =2 k-|V(G)| \geq 0 .
\end{aligned}
$$

Thus $\left|\mathcal{C}_{4} \cup \mathcal{C}_{5}\right|-\left|\mathcal{N}_{4}\right|-\left|\mathcal{N}_{5}\right| \geq 0,\left|\mathcal{C}_{4}\right|-\left|\mathcal{N}_{4}\right| \geq 0$ and $\left|\mathcal{C}_{5}\right|-\left|\mathcal{N}_{5}\right| \geq 0$. Hence Ann can follow the same strategy as given in Case 1 for presenting the vertices in $V_{4} \cup V_{5}$ to yield a winning strategy.

Case 3 : (iii) holds.

For $\left|\mathcal{C}_{4} \cup \mathcal{C}_{5}\right|-\left|\mathcal{N}_{4}\right|-\left|\mathcal{N}_{5}\right|=0$, Ben should have colored the vertices of $V_{3}$ with exactly $\left\{\left|\mathcal{C}_{4} \cup \mathcal{C}_{5}\right|-\left|\mathcal{N}_{4}\right|-\left|\mathcal{N}_{5}\right|\right\}^{*}=k-\left|V_{4}\right|-\left|V_{5}\right|$ colors which are given to the vertices of $V_{1}$. As observed in Case 2, $\left|\mathcal{C}_{4}\right|-\left|\mathcal{N}_{4}\right| \geq 0$ and $\left|\mathcal{C}_{5}\right|-\left|\mathcal{N}_{5}\right| \geq 0$. Thus Ann follow the same strategy as given in Case 1 for presenting the vertices in $V_{4} \cup V_{5}$ to yield a winning strategy. Next for $i=2,3,\left|\mathcal{C}_{i}\right|-\left|\mathcal{N}_{i}\right| \geq 0$. Since $\left\{c(v): v \in V_{3}\right\} \cap\left\{c(v): v \in V_{4}\right\}=\emptyset$ and $\left|\left\{c(v): v \in V_{1}\right\} \cap\left\{c(v): v \in V_{3}\right\}\right|=k-\left|V_{4}\right|-\left|V_{5}\right|$, we have $\mid\left\{c(v): v \in V_{4}\right\} \cap\{c(v): v \in$ $\left.V_{1}\right\}|=| V_{1}\left|-\left\{\left|\mathcal{C}_{4} \cup \mathcal{C}_{5}\right|-\left|\mathcal{N}_{4}\right|-\left|\mathcal{N}_{5}\right|\right\}^{*}=\right| V_{1} \mid-\left(k-\left|V_{4}\right|-\left|V_{5}\right|\right)$. As observed in Case 2, the value of $\left|\mathcal{C}_{2} \cup \mathcal{C}_{3}\right|-\left|\mathcal{N}_{2}\right|-\left|\mathcal{N}_{3}\right|$ reduces by 1 every time Ben colors a vertex in $V_{4}$ with a color given to a vertex in $V_{1}$. Now,

$$
\begin{aligned}
\left|\mathcal{C}_{2} \cup \mathcal{C}_{3}\right|-\left|\mathcal{N}_{2}\right|-\left|\mathcal{N}_{3}\right| & =\left\{\left|\mathcal{C}_{2} \cup \mathcal{C}_{3}\right|-\left|\mathcal{N}_{2}\right|-\left|\mathcal{N}_{3}\right|\right\}^{*}-\left|\left\{c(v): v \in V_{4}\right\} \cap\left\{c(v): v \in V_{1}\right\}\right| \\
& =k-\left|V_{2}\right|-\left|V_{3}\right|-\left(\left|V_{1}\right|-\left(k-\left|V_{4}\right|-\left|V_{5}\right|\right)\right) \\
& =2 k-|V(G)| \geq 0 .
\end{aligned}
$$

Thus again Ann can follow the same strategy as given in Case 1 for presenting the uncolored vertices in $V_{2} \cup V_{3}$ to yield a winning strategy.

Hence $G$ is $k$-indicated colorable for all $k \geq \chi(G)$.

Corollaries 3.7, 3.8, 3.9, 3.10 and Theorem 3.11 are some of the consequences of Theorem 3.6 .

Corollary 3.7 For $1 \leq i \leq 5$, let $m_{i}$ 's be positive integers. Then for the graph $G=$ $\mathbb{K}\left[C_{5}\right]\left(m_{1}, m_{2}, m_{3}, m_{4}, m_{5}\right), \chi(G)=\max \left\{\omega(G),\left\lceil\frac{|V(G)|}{2}\right\rceil\right\}$.

Proof. We know that, $\chi(G) \geq \max \left\{\left\lceil\frac{|V(G)|}{2}\right\rceil, \omega(G)\right\}$. If one closely observes Theorem 3.6, it can be seen that $G$ is $k$-indicated colorable for $k=\max \left\{\omega(G),\left\lceil\frac{|V(G)|}{2}\right\rceil\right\}$. Thus 
$\chi(G) \leq \chi_{i}(G) \leq \max \left\{\omega(G),\left\lceil\frac{|V(G)|}{2}\right\rceil\right\}$

An immediate consequence of Corollary 3.7 is one of the results proved by J. L. Fouquet et.al. in [6]. Namely, for $G=\mathbb{K}\left[C_{5}\right](m, m, m, m, m), m \geq 1, \chi(G)=\left\lceil\frac{5 m}{2}\right\rceil=\left\lceil\frac{|V(G)|}{2}\right\rceil$.

Corollary 3.8 If $G$ is a $\left\{P_{5}, C_{4}\right\}$-free graph, then $G$ is $k$-indicated colorable for all $k \geq \chi(G)$.

Proof. By Theorem 2.6, it is enough to prove the result for a connected $\left\{P_{5}, C_{4}\right\}$-free graph. Let $G$ be such a graph. Then by Theorem 3.4, $V(G)=V_{1} \cup V_{2}$ such that

(i) $\left\langle V_{1}\right\rangle$ is a $P_{5}$-free graph which is also chordal.

(ii) If $V_{2} \neq \emptyset$, then $\left\langle V_{2}\right\rangle=A_{1} \cup A_{2} \cup \cdots \cup A_{l}$ where each $A_{i}$ is a $\mathbb{K}\left[C_{5}\right]$, for every $i \in$ $\{1,2, \ldots, l\}$ for some $l \geq 1$. Also, $\left\langle N\left(A_{i}\right)\right\rangle$ is a complete subgraph of $V_{1}$ and $\left[A_{i}, N\left(A_{i}\right)\right]$ is complete.

Let the color set be $\{1,2, \ldots, k \geq \chi(G)\}$. Since $\left\langle V_{1}\right\rangle$ is chordal, $\operatorname{col}\left(\left\langle V_{1}\right\rangle\right)=\omega\left(\left\langle V_{1}\right\rangle\right) \leq k$. By Theorem 3.1, Ann has a winning strategy for the vertices of $V_{1}$ using $k$ colors. Let Ann follow this winning strategy for presenting the vertices of $V_{1}$. Let $Q_{i}=\left\langle N\left(A_{i}\right)\right\rangle \subseteq V_{1}$, for every $i \in\{1,2, \ldots, l\}$. Clearly $\chi\left(A_{i}+Q_{i}\right)=\chi\left(A_{i}\right)+\chi\left(Q_{i}\right) \leq \chi(G) \leq k$. Thus $k-\chi\left(Q_{i}\right) \geq \chi\left(A_{i}\right)$, for $1 \leq i \leq l$. By Theorem 3.6, each $A_{i}$ is $k$-indicated colorable for every $k \geq \chi\left(A_{i}\right)$ and hence Ann has a winning strategy for each $A_{i}$ while using $k-\chi\left(Q_{i}\right) \geq \chi\left(A_{i}\right)$ colors, for $1 \leq i \leq l$. Since $A_{i}$ 's are disjoint, if Ann presents the vertices of $A_{i}$ 's for $1 \leq i \leq l$ by using these winning strategies, Ben cannot create a blocked vertex. Thus $G$ is $k$-indicated colorable for all $k \geq \chi(G)$.

Recall that a graph $G$ is said to be a split graph, if $V(G)$ can be partitioned in to two subsets such that the subgraph induced by one set is a clique and the other is totally disconnected.

Corollary 3.9 Let $S_{1}, S_{2}, S_{3}, S_{4}, S_{5}$ be the split graphs. The graph $G=C_{5}\left(S_{1}, S_{2}, S_{3}, S_{4}, S_{5}\right)$ is $k$-indicated colorable for all $k \geq \chi(G)$.

Proof. Let $G$ be the graph $C_{5}\left(S_{1}, S_{2}, S_{3}, S_{4}, S_{5}\right)$. For $1 \leq i \leq 5, V\left(S_{i}\right)=V_{i} \cup U_{i}$, where $\left\langle V_{i}\right\rangle$ is a maximum clique and $\left\langle U_{i}\right\rangle$ is an independent set respectively. Let the color set be $\{1,2, \ldots, k \geq \chi(G)\}$. By Theorem 3.6, there is a winning strategy for the subgraph $C_{5}\left(V_{1}, V_{2}, V_{3}, V_{4}, V_{5}\right)$ of $G$ using $k$ colors. Let Ann follow this winning strategy to present the vertices of $C_{5}\left(V_{1}, V_{2}, V_{3}, V_{4}, V_{5}\right)$. Next, let Ann presents the remaining vertices of $G$, namely the vertices in $\cup_{i=1}^{5} U_{i}$, in any order. Since each of the vertex $x \in U_{i}, 1 \leq i \leq 5$, has a non neighbor in $V_{i}$, the color of that non neighbor in $V_{i}$ will be available for $x$. Thus Ben cannot 
create any blocked vertex and hence Ann wins the game on $G$ with $k$ colors.

An immediate consequence of Theorem $3.2,3.5$ and Corollary 3.9 is Corollary 3.10 ,

Corollary 3.10 If $G$ is connected $\left\{P_{5},\left(\overline{P_{2} \cup P_{3}}\right), \overline{P_{5}}\right.$, Dart $\}$-free graph that contains an induced $C_{5}$, then $G$ is $k$-indicated colorable for all $k \geq \chi(G)$.

Now let us consider the indicated coloring of connected $\left\{P_{2} \cup P_{3}, C_{4}\right\}$-free graphs.

Theorem 3.11 If $G$ is a connected $\left\{P_{2} \cup P_{3}, C_{4}\right\}$-free graph, then $G$ is $k$-indicated colorable for all $k \geq \chi(G)$.

Proof. Let $G$ be a connected $\left\{P_{2} \cup P_{3}, C_{4}\right\}$-free graph. By Theorem 1 , if $G$ is chordal then $\operatorname{col}(G)=\omega(G)=\chi(G)$. By Theorem 3.1, $G$ is $k$-indicated colorable for all $k \geq \chi(G)$. Suppose $G$ is not chordal, then there exists a partition $\left(V_{1}, V_{2}, V_{3}\right)$ of $V(G)$ such that $\left\langle V_{1}\right\rangle \cong$ $\overline{K_{m}}$ for some $m \geq 0,\left\langle V_{2}\right\rangle \cong K_{t}$ for some $t \geq 0$ and $\left\langle V_{3}\right\rangle \cong G_{i}$ for some $i, 1 \leq i \leq 17$ (see Figure 3). Let us divide the proof into two cases as follows.

Case $1: V_{2}=\emptyset$

Since $\left[V_{1}, V_{3}\right]=\emptyset$ and $G$ is connected, $G \cong G_{j}$ for some $j, 1 \leq j \leq 17$. Hence it is enough to show that for $1 \leq j \leq 17, G_{j}$ is $k$-indicated colorable for all $k \geq \chi\left(G_{j}\right)$. Let us first consider the $G_{j}$ 's when $j \in\{1,2, \ldots, 17\} \backslash\{6,7,8,9\}$. It is not difficult to observe that for these $j \in\{1,2, \ldots, 17\} \backslash\{6,7,8,9\}, \operatorname{col}\left(G_{j}\right)=\chi\left(G_{j}\right)$. Hence by Theorem $3.1, G_{j}$ is $k$-indicated colorable for all $k \geq \chi\left(G_{j}\right)$.

For $j \in\{6,7,8,9\}$, it can be seen that $\operatorname{col}\left(G_{j}\right) \neq \chi\left(G_{j}\right)$, so we consider these graphs separately. The graph $G_{6} \cong C_{6}$ and hence $k$-indicated colorable for all $k \geq \chi\left(G_{6}\right)$. The graph $G_{7} \cong \mathbb{K}\left[C_{5}\right]\left(m_{1}, m_{2}, m_{3}, m_{4}, m_{5}\right)$, where each $m_{i} \geq 1,1 \leq i \leq 5$ and hence by Theorem 3.6 , $G_{7}$ is $k$-indicated colorable for all $k \geq \chi\left(G_{7}\right)$. Next the graph $G_{8} \cong P$, the Petersen graph. In [13], it has been showed that the Petersen graph $P$ is $k$-indicated colorable for all $k \geq \chi(P)$. Finally, let us consider the graph $G_{9}$. It is easy to check that $\chi\left(G_{9}\right)=3$ and $\operatorname{col}\left(G_{9}\right)=5$. By Theorem 3.1, it is enough to show that $G_{9}$ is 3 and 4-indicated colorable. Let us first consider $G_{9}$ with 3 colors, namely $\{1,2,3\}$. If Ann presents the vertices of $G_{9}$ in the order $p, q, r, s, t, u, v, w, x$, then Ben always has an available color for each of the vertices. Now let us consider $G_{9}$ with 4 colors, namely $\{1,2,3,4\}$. Let Ann start by presenting the vertices $p, q, r$. Without loss of generality, let Ben color these vertices with 1, 2 and 3 respectively. Now let Ann present the vertex $u$. Suppose Ben colors $u$ with 1 or 4 , then Ann will presents the remaining vertices in the order $s, t, v, x, w$. Suppose Ben colors $u$ with 2 or 3 , then Ann 
will presents the remaining vertices in the order $w, x, v, t, s$. This guarantees the fact that Ben cannot block any of the vertex. Thus Ann wins the game with 4 colors.

Case $2: V_{2} \neq \emptyset$

Recall that $\left\langle V_{2}\right\rangle \cong K_{t}$ and $\left\langle V_{3}\right\rangle \cong G_{j}, 1 \leq j \leq 17$. Since $V_{1}$ is independent and $\left[V_{1}, V_{3}\right]=$ $\emptyset$, we can color the vertices of $V_{1}$ with one of the colors of $V_{3}$. Thus $\chi(G)=\chi\left(K_{t}+G_{j} \backslash S\right)=$ $t+\chi\left(G_{j} \backslash S\right)$, for $j \in\{1,2, \ldots, 17\}$. Let us first consider the graphs $G$ for which $\left\langle V_{3}\right\rangle \cong G_{j}$, $j \in\{1,2,3,4,5\}$, the graph with $S \neq \emptyset$. Without much difficulty one can show that $\operatorname{col}(G)=$ $\chi(G)$. Thus by Theorem 3.1, $G$ is $k$-indicated colorable for all $k \geq \chi(G)$.

Next, let us consider the graphs $G$ for which $\left\langle V_{3}\right\rangle \cong G_{j}, j \in\{6,7, \ldots, 17\}$. We know that, $K_{t}$ is $k_{1}$-indicated colorable for all $k_{1} \geq t$ and by Case $1, G_{j}$ is $k_{2}$-indicated colorable for all $k_{2} \geq \chi\left(G_{j}\right), j \in\{6,7, \ldots, 17\}$. Hence by Theorem 3.2, we see that $\left\langle V_{2} \cup V_{3}\right\rangle \cong K_{t}+G_{j}$ is $k$-indicated colorable for all $k=k_{1}+k_{2} \geq t+\chi\left(G_{j}\right)=\chi(G)$ and hence Ann has a winning strategy for $\left\langle V_{2} \cup V_{3}\right\rangle$ while using $k$ colors for any $k \geq \chi(G)$. Next, let Ann present the vertices of $V_{1}$ in any order. Since $V_{1}$ is independent and $\left[V_{1}, V_{3}\right]=\emptyset$, the colors used in $V_{3}$ are available to Ben for each vertex in $V_{1}$. Thus $G$ is $k$-indicated colorable for all $k \geq \chi(G)$.

\section{Acknowledgment}

For the first author, this research was supported by the Council of Scientific and Industrial Research, Government of India, File no: 09/559(0096)/2012-EMR-I. Also, for the third author, this research was supported by the UGC-Basic Scientific Research, Government of India.

\section{References}

[1] N. R. Aravind, T. Karthick, C. R. Subramanian, Bounding $\chi$ in terms of $\omega$ and $\Delta$ for some classes of graphs, Discrete Math. 311 (2011) 911-920.

[2] G. Bacsó, Z. Tuza, Dominating cliques in $P_{5}$-free graphs, Period. Math. Hung. 21 (1990) 303-308.

[3] T. Bohman, A. Frieze, B. Sudakov, The game chromatic number of random graphs, Random Structures and Algorithms, 32 (2008) 223-235.

[4] A. Brandstädt, R. Mosca, On the structure and stability number of $P_{5^{-}}$and co-chair-free graphs, Discret. Appl. Math. 132, (2004) 47-65.

[5] S. A. Choudum, T. Karthick, Maximal cliques in $\left\{P_{2} \cup P_{3}, C_{4}\right\}$-free graphs, Discrete Math. 310 (2010) 3398-3403.

[6] J. L. Fouquet, V. Giakoumakis, F. Maire, H. Thuillier, On graphs without $P_{5}$ and $\overline{P_{5}}$, Discrete Math. 146 (1995) 33-44. 
[7] S. Francis Raj, R. Pandiya Raj, H. P. Patil, On indicated chromatic number of graphs, Graphs and Combin. 33 (2017) 203-219.

[8] A. Grzesik, Indicated coloring of graphs, Discrete Math. 312 (2012) 3467-3472.

[9] D. Guan, X. Zhu, The game chromatic number of outerplanar graphs, J. Graph Theory, 30 (1999) $67-70$

[10] P. V. Hof, D. Paulusma, A new characterization of $P_{6}$-free graphs, Discrete Appl. Math. 158 (2010) 731-740.

[11] M. Lasoń, Indicated coloring of matroids, Discrete Appl. Math. 179 (2014) 241-243.

[12] J. Liu, Y. Peng, C. Zhao, Characterization of $P_{6}$-free graphs, Discrete Appl. Math. 155 (2007) 1038-1043.

[13] R. Pandiya Raj, S. Francis Raj, H. P. Patil, On indicated coloring of graphs, Graphs and Combin. 31 (2015) 2357-2367.

[14] B. Randerath, I. Schiermeyer, 3-colorability $\in P$ for $P_{6}$-free graphs, Discrete Appl. Math. 136 (2004) 299-313.

[15] Y. Sekiguchi, The game coloring number of planar graphs with a given girth, Discrete Math. 330 (2014) 11-16.

[16] D. P. Sumner, Subtrees of a graph chromatic number, The Theory and Applications of Graphs, G. Chartrand (Ed.), John Wiley, New York, 1981.

[17] D. B. West, Introduction to Graph Theory, vol. 2. Prentice-Hall, Englewood Cliffs (2000).

[18] J. Wu, X. Zhu, Lower bounds for the game colouring number of partial $k$-trees and planar graphs, Discrete Math., 308 (2008) 2637-2642.

[19] X. Zhu, The game coloring number of planar graphs, J. Combin. Theory Ser. B, 75 (1999) 245258. 